\title{
El reto de la gestión humana frente a la complejidad y pluralidad cultural
}

\author{
Rodríguez Pérez, María Candelaria*
}

\section{Resumen}

La diversidad se traduce en la diferenciación que parte de la condición de género, etnicidad, religión, clase social, habilidad física, orientación sexual y edad. Estas orientaciones emanan producto de los cambios que se ha dado a partir de la globalización e impacto de las tecnologías de la información y comunicación. En consecuencia se asume, que la fuerza laboral tiene carácter diferenciado y plural, que requiere de mecanismos que permitan lograr administrar la diversidad y la cultura como un elemento condicionante y de impacto en las nuevas relaciones laborales y en la gestión de lo humano bajo esquemas de complejidad. El presente artículo tiene como objetivo indagar sobre la complejidad, diversidad, pluralidad y cultura, para establecer algunos de los retos que emergen en las formas de gestionar lo humano. La metodología que se utilizó, fue el análisis documental a través de la triangulación de información sobre las categorías asumidas, para lograr la construcción de una reflexión en relación al tema. La conclusión se enmarcó en la necesidad de lograr fomentar una gestión humana con actitud pluralista, abierta, flexible y de gran sensibilidad transcultural, orientada a la búsqueda de la igualdad de oportunidades y el trato digno del ser humano.

Palabras clave: Gestión humana, pluralismo cultural, cultura, complejidad, hipercomplejidad y diversidad.

\section{The Challenge of Human Management in the Face of Cultural Complexity and Plurality}

\author{
Abstract \\ Diversity translates into differentiation that begins with conditions of gender, ethnicity, relig- \\ ion, social class, physical ability, sexual orientation and age. These orientations have come out as \\ Recibido: 14-02-07. Aceptado: 26-06-08 \\ * $\quad$ Lic. en Relaciones Industriales, Especialista en Gerencia de Recursos Humanos y Magíster en \\ Administración del Trabajo y Relaciones Laborales y candidato a doctor en el Programa de \\ Doctorado en Ciencias Administrativas de la UNESR. Profesor Asociado a dedicación exclusi- \\ va de la Cátedra de Sociología General de la Escuela de Relaciones Industriales de la Facultad \\ de Ciencias Económicas y Sociales de la Universidad de Carabobo. \\ E-mail: maotaiza@cantv.net; marycande65@hotmail.com
}


a product of the changes that have occurred with globalization and the impact of information and communication technologies. As a consequence, it is assumed that the labor force has a differentiated and plural character which requires mechanisms that permit administering diversity and culture as a conditioning element that impacts new labor relations and human management under perceptions of complexity. The objective of this article is to investigate complexity, diversity, plurality and culture, to establish some of the challenges that emerge in ways of managing human beings. The methodology used was documentary through triangulation of the information about assumed categories, to construct a reflection related to the theme. The conclusion was framed by the need to foment human management with a pluralist, open, flexible attitude that has great transcultural sensitivity, oriented toward a search for the equality of opportunities and treatment worthy of the human being.

Key words: Human management, cultural pluralism, culture, complexity, hyper-complexity and diversity.

\section{Introducción}

La complejidad de los fenómenos sociales es uno de los rasgos predominante que caracteriza el escenario actual. Por esta razón, la misma se ha convertido en la categoría de análisis de las realidades sociales bajo el contexto emergente. De allí que hablar de complejidad, denota en cierta medida la "incapacidad de comprensión, ya que el objeto de estudio parece desbordar al investigador intelectualmente" Navarro (1996:2). La justificación parte, al decir de Morin (1998:132) de que la complejidad esta presente en el "tejido de eventos, acciones, interacciones, retroacciones, determinaciones y azares que constituyen el mundo fenoménico." En consecuencia, se esta en presencia de vínculos sociales caracterizados por la pluralidad, diversidad y diferenciación en distintos planos: el social, organizacional, económico, político, cultural e individual, que hace que el enfoque que se adopte sea multicausal, transdisciplinar e integrador razón por la cual rebasa la capacidad de análisis.
Siendo así, la gestión humana en las organizaciones como realidad fenoménica, no debe perder de vista el efecto de la complejidad en razón de las implicaciones que conllevan a enfrentar mayor diversidad y pluralidad cultural como consecuencia de un mundo más relacionado, interconectado, abierto y flexible.

No obstante, el plantear la necesidad de administrar la diversidad y alcanzar una actitud plural, responde a las desviaciones que se han venido dando en la sociedad y en las organizaciones y las cuales se traducen en prácticas de intolerancia, exclusión, discriminación y etnocentrismo, entre otras. Por ende, en un mundo que tiende a conciliar estos errores del pasado y de la historia, se motiva el análisis y discusión acerca del tratamiento de estos temas.

En tal sentido, el ensayo pretende desde las consideraciones del paradigma de la complejidad, inferir los rasgos de la pluralidad cultural y diversidad y así establecer algunos de los retos que emergen en las formas de gestionar lo humano, logrando con ello contribuir con una visión integrada y vinculante entre las categorías asumidas en el tratamiento del tema. 
El reto de la gestión humana frente a la complejidad y pluralidad cultural Rodríguez Pérez, María Candelaria

Para el logro del objetivo, el ensayo se estructuró de la siguiente manera: Un abordaje inicial que define algunos de los aspectos sobre la complejidad y sus aristas concretadas en la pluralidad y diversidad, en este punto se marcan las relaciones entre las categorías asumidas en el documento. Enseguida se desarrollo los argumentos que posicionan a la gestión humana en el paradigma de la complejidad y se resaltan los roles a desarrollar como parte de los retos de administrar de diversidad y el pluralismo cultural.

\section{Aproximación a la definición de complejidad}

Uno de los determinismos presentes en la sociedad de hoy, es el referido a la complejidad de los fenómenos sociales, que emerge del objeto y sujeto, en un interjuego que desborda la capacidad intelectual en la búsqueda por querer aproximarse a una definición de la realidad, ya que hay la coexistencia, de una multitud de elementos y numerosísimas relaciones entre los mismos Navarro (1996). Sin embargo, la complejidad viene dada por el carácter emergente y dinámico de los contextos y en la capacidad para generar en determinada realidad, elementos nuevos -emergentes- y nuevas relaciones -también emergentes- entre esos elementos.

En contraste a las ideas anteriores, Agudelo y Alcala (2003) establecen un conjunto de propiedades inherentes a la definición de complejidad y las cuales se esbozan a continuación:

- La complejidad se da en los sistemas:

Un elemento aislado no puede au- mentar su complejidad porque no interactúa con otros elementos endónenos y exógenos.

- La complejidad es un proceso evolutivo, no sigue una ecuación lineal.

- La ley de la complejidad establece que en todo sistema, la complejidad de su estructura es directamente proporcional a la información de las leyes que rigen su funcionamiento y que dicha estructura puede contener. Se reafirma aquí la información y comunicación son factores de complejización de los sistemas.

- La complejidad se inicia con un sistema de mínima complejidad y avanza o evoluciona hacia una mayor complejidad. Esto tiene que ver con el carácter emergente, evolutivo que encierra consigo rasgos tales como "lo enredado, lo inextricable, la ambigüedad y la incertidumbre" (Morin, 1998).

En conclusión y parafraseando a Agudelo y Alcala (2003) un sistema es capaz de alcanzar cierto grado de complejidad al ocurrir un evento crítico que inicia el desorden del sistema el cual decae hasta el punto de decisión, cuando surgen tres alternativas: su evolución, el estancamiento del sistema o su extinción.

Asimismo, es meritorio resaltar que en la sociedad hay dos tipos de realidades, una que marca a las sociedades humanas como culturas materiales, ya que modifican el entorno ecológico de manera profunda, el cual crea un ecosistema en buena medida artificial, y por otra parte, sociedades humanas como realidades de conciencia, alojadas en la mente de los individuos que la integran. Lo que permite suponer la presencia de un mun- 
do cultural e ideológico diverso, en donde los valores y creencias del individuo son permanentemente cambiantes, lo cual genera una hipercomplejidad que abarca a todas las estructuras sociales, desplegando una variabilidad cada vez más acelerada en un proceso de diferenciación constante, estableciendo toda una dinámica de incertidumbre que se expande bajo el signo y las interacciones virtuales, basadas en información y conocimiento, lo que determina un contexto que busca explicarse a través de imaginarios, proyecciones y predicciones del futuro basadas en la reconstrucción del pasado (Navarro, 1996).

Por tanto, la complejidad se convierte en un escenario incierto, difícil de definir y dibujar, ya que sus fronteras son borrosas y son siempre superpuestas (Morin, 1998). Por ende se supone, que la profundización en el estudio de los fenómenos sociales, debe escapar de la racionalidad lógica para entrar en la dialógica que concilia lo complementario y antagónico para poder aproximarse a un análisis integral del fenómeno.

\section{Diversidad y pluralidad. Aristas de la complejidad}

Como ya se dijo en líneas anteriores, la complejidad implica elementos, estructuras, relaciones y sistemas que emergen de los ya existentes y como resultado de los cambios impulsados por la globalización y la incorporación tecnológica a los procesos, añaden cambios a considerar en la indagación de los fenómenos sociales. No obstante, es necesario introducir algunos elementos de enlace entre la complejidad, diversidad y plu- ralidad. Con este fin, se desarrollan a continuación los argumentos que sustentan las aristas de la complejidad:

a)Sistemas sociales complejos o hipercomplejos como eje del enfoque.

El ámbito de la disertación se ubica en el plano de lo social, justificado en el hecho de un sujeto como actor social, el cual activa y promueve los cambios, pero a su vez esta influenciado por los mismos, de manera que a efecto del presente ensayo, la complejidad se enmarcó en los sistemas sociales.

Por otro lado, se toma la categoría planteada por Morin (1995:174) referida a la hipercomplejidad como enfoque complementario, ya que la misma se "corresponde con el debilitamiento o desaparición de los principios rígidos de programación, de jerarquización, de especialización en beneficio de estrategias creativas o inventivas, de la polivalencia funcional, del policentrismo en el control y en la decisión ". Concretando, el autor refuerza la idea de los cambios en los supuestos que han prevalecido y que llevan a las sociedades a transitar desde lo complejo hasta lo hipercomplejo. No obstante, es importante retomar la idea inicial que rescata la intención de concentrase en el sistema social como eje del enfoque de la reflexión y que las categorías esbozadas son tomadas como elementos vinculantes desde la teoría.

b)Sistema cultural como punto de enlace

El desarrollo de los sistemas sociales en complejos o hipercomplejos, revelan una deconstrucción del modo de sociabilidad humana, de las formas de es- 
El reto de la gestión humana frente a la complejidad y pluralidad cultural Rodríguez Pérez, María Candelaria

tructuras y organización, de los nichos de poder, de las formas de conciencia social, los valores y creencias. En tal sentido, se enlaza lo social con lo cultural, amalgamados dichos sistemas en una unión de interdependencia, en la cual se contienen en un todo. Por otro lado, si se afirma que los sistemas sociales tienden a la complejidad e hipercomplejidad, es necesario asumir el impacto que se origina en el sistema cultural.

c) La diversidad como característica de los sistemas sociales y culturales

Por consecuencia de las consideraciones anteriores, se reconoce la diversidad como condición y característica de los sistemas sociales y culturales. La misma se define como "el grado de las diferencias humanas básicas en un grupo dado, las cuales están representadas en el género, etnicidad, religión, clase social, habilidad física, orientación sexual y edad" (Certo, 2001:528). En otras palabras, la diversidad es producto de la diferenciación social y cultural.

\section{La pluralidad como mecanismo de reconocimiento de las diferencias}

La coexistencia de los sistemas sociales y culturales depende de su capacidad de adaptación, tolerancia e inclusión de las distintas culturas que subyacen en un mismo entorno. Daft y Marcic (2005) establecen que el pluralismo significa que una organización acoge y aglutina varias subculturas, logrando aceptar diferentes formas de pensamiento y comportamiento.

Por esta razón, la diversidad parte de las diferencias individuales y la pluralidad es la intención de aceptar la diversidad para lograr la integración. Por ende, la diversidad y pluralidad emanan del individuo y los grupos sociales, de su condición y naturaleza. Asimismo las aristas de la complejidad matizadas del carácter plural y diferenciado, pone su acento en "el sistema cultural”, ya que encierra los determinismos producto de la diversidad que emana del carácter emergente de los sistemas.

A fin de lograr la adopción de una perspectiva de pluralismo, Daft y Marcic (2005) plantean tres pasos, que se presentan a continuación:

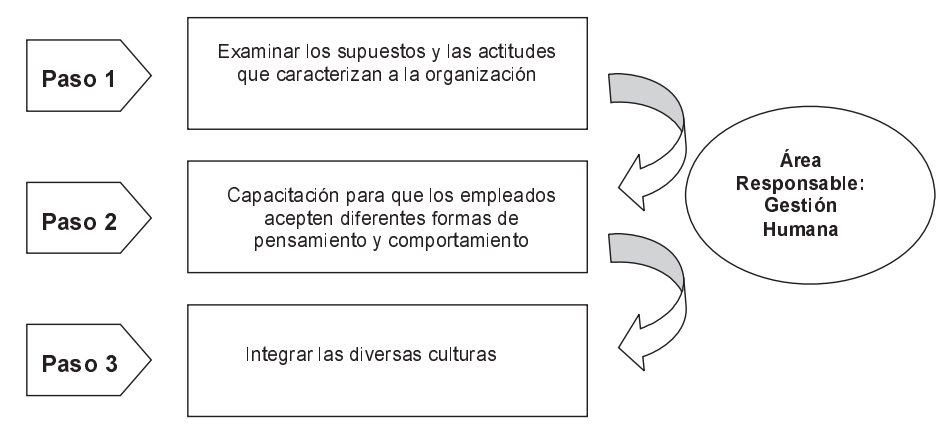

Fuente: Elaboración propia tomado de Daft y Marcic (2005:343). 


\section{Algunas consideraciones sobre la cultura y la cultura organizacional}

Hasta ahora, se ha ido especificando los aspectos referidos a la complejidad, diversidad y pluralidad, no obstante el eje transversal de la reflexión se enmarca en la cultura como categoría. De manera que si se profundiza en este aspecto, se tendrá más clara las connotaciones e impactos que la misma tiene en el contexto social y en específico, en el de las organizaciones.

Por tanto, es pertinente traer a consideración la clasificación a la que responde la cultura, de manera que se tienen dos categorías: cultura material e inmaterial. La primera, esta compuesta por el sistema ideológico de toda sociedad, en donde se encuentran los valores, sistema de creencias, ideas y filosofía; y el sistema sociológico, en donde están las pautas que permiten ordenar la sociedad a través de normas, costumbres, roles, estatus entre otros. La segunda, referida a la cultura inmaterial, viene dada por aquello que el hombre inventa y crea para satisfacer sus necesidades y en ella esta inmersa la innovación tecnológica (Arias, 2000:287-293).

En tal sentido se puede deducir, que la cultura signada por los procesos de transformación del contexto emergente, bulle y cambia de estado para convertirse en el vapor de los intangibles del mundo de hoy. En cuanto a que están representados en los valores predominantes, en los imaginarios, en la simbología, en los iconos, en las representaciones sociales, en los determinismos culturales conjugados en la diversidad, en el conoci- miento, en las lealtades y en el lenguaje entre otros.

Se entra en el mundo del significado, en donde se administra desde el sentido intuitivo y semiótico, para descifrar y orientar las acciones bajo una racionalidad interpretativa, que amerita considerar la pluralidad cultural y la diversidad en todas las relaciones que acontecen en la organización. En consecuencia, administrar lo humano lleva consigo el reto de gestionar intangibles tales como el conocimiento, lealtades, experiencias, talento y valores entre otros.

Por otra parte Morin (1995:143), expresa una posición diferente orientada a la masificación de la cultura por el hecho de que la misma está "constituida por una enorme cantidad de información, que crece sin cesar, pero que se destruye sin cesar, convirtiéndose en ruido, dando paso a una superficialidad en el modo de análisis, como resultado del carácter efímero que posee.

Por consecuencia, se conduce a dos planos claramente esbozados: uno, el de la complejidad que emerge como fruto del espacio y tiempo liberados en un mundo laboral flexible, de organizaciones fractales y de actores considerados ciudadanos del mundo. $Y$ el otro, como resultado de la incorporación de las tecnologías de la información y comunicación a muchos de los procesos, lo cual desborda de información y se traduce en una cultura virtual, en un espacio paralelo al real, que también pluraliza y da complejidad tanto en el modo de hacer como en el sistema de creencias imperante en la sociedad y en las organizaciones que la componen. 
El reto de la gestión humana frente a la complejidad y pluralidad cultural Rodríguez Pérez, María Candelaria

A pesar de las consideraciones expuestas, la cultura de masas y el carácter ingrávido y plural de la misma, gesta una nueva forma de funcionar, de hacer, de pensar y creer, impactando las formas de vida y de trabajo del hombre. Se esta en presencia de la necesidad de reorientar la gestión humana hacia la inclusión, superando estereotipos, discriminación, etnocentrismo y prejuicios, en función de los factores que marcan la diversidad y pluralidad bajo parámetros culturales diferenciados.

Por esta razón, desde el plano de las organizaciones y del mundo del trabajo, se plantea una realidad distinta a la que operaba en la sociedad moderna, por lo cual es imperioso construir puentes que permitan alcanzar "estados de fusión" de la cultura al interno de estructuras locales, respetando la pluralidad y diferenciación de la misma.

Por otro lado, se clasifica la cultura como componente de la filosofía de toda organización y es tomada como un mecanismo "activo y movilizador de la organización (...) es un marco de referencia compartido; son valores aceptados por el grupo de trabajo que indica cuál es el modo esperado de pensar y actuar frente a situaciones concretas" (Etkin y Schvarrstein ,1997:204).

En concreto, según Etkin y Schvarrstein (1997) y en relación con las formas de administrar la diversidad y pluralismo se puede suponer lo siguiente:

En el marco de la cultura organizacional, las pautas compartidas son desde el punto de vista racional, como información a procesar en el momento de resolver los actos individuales. Pero además de sus componentes racionales y tangibles, los rasgos culturales también se instituyen en términos emocionales y se viven como dramas, crisis o dilemas en el grupo social. Como construcción percibida en el nivel de los participantes, la cultura permite incorporar elementos reflexivos para enfrentar la crisis y, de esta manera, enmarcar las fantasías y el modo imaginario como la organización es vivida por sus participantes.

Así es que, el desarrollo de una cultura organizacional con base al respeto de las diferencias, la aceptación de las minorías, la inclusión, el pluralismo, la igualdad y la equidad, es un reto evidente de la gestión humana. Después de todo, es a través de la institucionalización de una cultura tolerante al interno de la organización, que se pueden construir políticas que le den soporte y permitan a los miembros, identificarse y adaptarse de manera más fácil y oportuna. Ya que si bien, la cultura organizacional puede permear relaciones de pluralidad y aceptación de la diversidad, por otro lado, puede convertirse en un obstáculo si no se han planificado estrategias en esta línea de acción.

\section{El pluriculturalismo y el reto de administrar la diversidad}

Como resultado a lo que se viene planteando, emerge uno de los retos actuales en la gestión humana, esto es, lograr políticas sociales y empresariales incluyentes y transculturales, formalizadas en mecanismos de gestión que permitan administrar la diversidad.

A fin de que se profundice en esta consideración, se toma como referencia la Declaración Universal de la UNESCO (2002) sobre diversidad cultural, en cuan- 
to reconoce en su artículo $1^{\circ}$ que "La cultura adquiere formas diversas a través del tiempo y del espacio. Esta diversidad se manifiesta en la originalidad y la pluralidad de las identidades que caracterizan los grupos y las sociedades que componen la humanidad." De manera que la diversidad y la pluralidad forman parte de los derechos a preservar en toda relación social y laboral. No se puede actuar en menoscabo del mismo.

A simple vista, desde el plano de las organizaciones, cada día se está dando con más frecuencia la necesidad de adoptar una posición orientada hacia la "pluralidad cultural", debido al efecto de la mundialización de las empresas como resultado de ubicar instalaciones en otros países y aprovechar mercados de trabajo determinados, además de la necesidad de rotar personal clave hacia otros países en áreas de competencias en donde quizás el país anfitrión en el que opera una filial, no cuenta con personal formado según los requerimientos y cultura de la empresa de origen.

Como consecuencia, se produce el multiculturalismo que genera diferencias en las identidades al interno de las organizaciones de corte mundial, impulsando a la necesidad de administrar esa diversidad y establecer políticas que permitan gestionar con actitud pluralista. En otras palabras "se tendrá que aprender a acomodar muchos factores culturales diferentes en las culturas de organizaciones particulares" (Stoner et al., 1996:210). En tal sentido, la pluralidad busca entonces "reconocer y aceptar las diferencias culturales, individuales y grupales" (Certo, 2001:542), constituía en un derecho más del sujeto.
En otras palabras, el pluriculturalismo hace referencia "a la existencia de muchos antecedentes y factores culturales que son importantes para las organizaciones, y también que las personas, a pesar de sus diferentes antecedentes pueden coexistir y prosperar en la organización" (Stoner et al., 1996: 208), impulsando a la convivencia, adaptación, tolerancia y aceptación de los seres humanos en sociedad y grupos.

Taylor Cox (tomado de Stoner et al., 1996:216) plantea seis argumentos que apuntan hacia la necesidad de administrar la diversidad como estrategia que permite, entre otros beneficios, el éxito de la organización. Parafraseando al autor, se establece que la administración de la diversidad cultural logra entre otras cosas:

- Mejorar los costos de la organización en relación con empresas que no emprendan acciones orientadas a manejar la pluralidad.

- Así mismo ayuda a dar una imagen que permite atraer mejor recurso humano, debido a que se trascienden los estereotipos generados por las diferencias culturales.

- Por otra parte, contar con personas de distintas culturas favorece a la perspicacia y sensibilidad necesaria para incursionar en distintos mercados, debido al conocimiento que tiene el personal expatriado de sus culturas de origen.

- Además permite el incentivo a la creatividad por el manejo de la diversidad, siendo otra de las ventajas que se obtienen al emprender la administración con criterios de pluriculturalismo. 
El reto de la gestión humana frente a la complejidad y pluralidad cultural Rodríguez Pérez, María Candelaria

- La heterogeneidad de los grupos, permite tener una perspectiva más compleja y diversa para solucionar los problemas, lo cual genera mejores decisiones.

- La administración de la diversidad flexibiliza a la organización para reaccionar frente a los cambios a los cuales ésta se enfrenta.

En concreto, Taylor Cox (tomado de Stoner et al., 1996:216), reafirma la importancia de administrar la diversidad en un ambiente cada vez más multicultural, sin embargo, el reto se presenta cuando hay que definir qué acciones hay que emprender para lograr coexistir, superando las diferencias y las barreras que se erigen como consecuencia de confluir personas de distintas raíces, sexo, creencias, valores, cultura y procedencia geográfica, en un entorno que supera a lo físico y trasciende incluso a lo virtual.

\section{Visión integral de las implicaciones de las categorías involucradas: Sistema social, sistema cultural, pluralidad, diversidad y gestión humana}

A continuación, se presenta una figura que integra las categorías de análisis adoptadas en el presente ensayo, con el fin de ilustrar las vinculaciones y consolidar la comprensión de las implicaciones y relaciones desde un nivel macro (social y cultural) hasta el micro (organización y cultura organizacional). Por otro lado se observa que el soporte en relación a la administración de la diversidad, pluralidad y cultura organizacional recae en la gestión humana. En el apartado siguiente se aborda las implicaciones y retos de la gestión humana en respuesta a estos cambios dados desde la perspectiva del paradigma de la complejidad.

Figura 1. La gestión humana y su relación con el sistema socio-cultural.

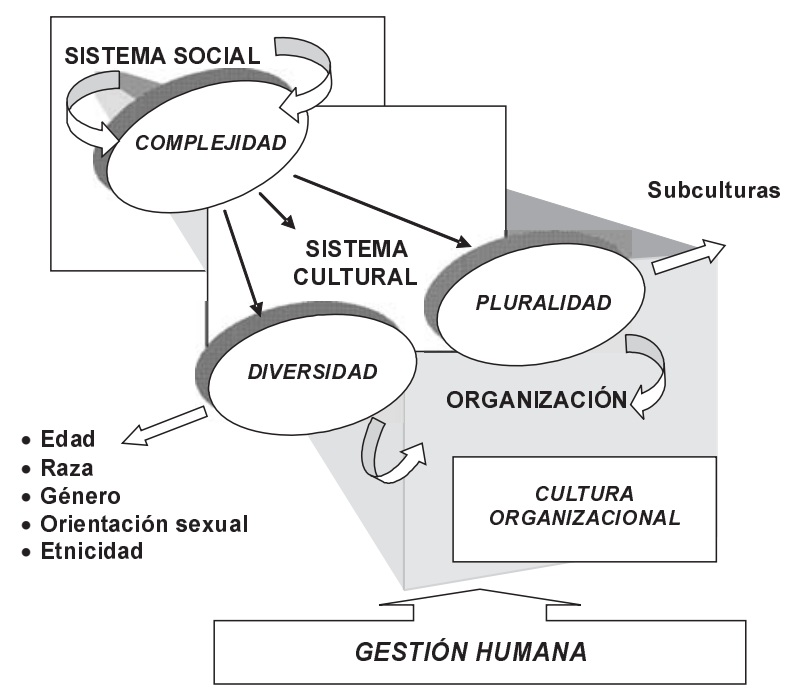




\section{El reto de la Gestión humana frente a la complejidad}

La gestiona humana, debe considerar al hombre de forma integral, en sus dimensiones mentales, sociales, culturales y emocionales, en tal sentido, se supera la concepción de trabajador, por la de colaborador en los procesos de trabajo. Así mismo, el hombre es considerado desde la perspectiva de un ser sistémico, el cual opina y es dueño de sus ideas, se autorregula, y esta conciente de la necesidad de prepararse y desarrollar competencias. Digamos, que no puede ser visto como recurso o capital, sino en su justa dimensión, la humana. En consecuencia, se esta en presencia de un fenómeno complejo, ya que por un lado, está el hombre en su dimensión humana e individual y por el otro, en su dimensión social y cultual, insertado en un organización.

Por lo tanto, basados en los argumentos precedentes, la Gestión Humana ha entrado en el paradigma de la complejidad, lo que implica suponer que se está en presencia de nuevos requerimientos que parten de las configuraciones del trabajo, caracterizadas por la especialización, intermediación de la tecnología, polivalencia y heterogeneidad, participación y solidaridad, entre otros, dando por superado el rol tradicional, para entrar en un rol que supone considerar los siguientes retos, entre los cuales se pueden mencionar:

- Administrar bajo esquemas de flexibilidad laboral

- Enmarcarse en el condicionante estratégico.
- Asumir un rol que abarque la complejidad y diferenciación funcional de las tareas.

- Enfrentar el reto de actores sociales con un grado de conocimiento más profundo y especializado el cual requiere de contratos diferenciados dirigidos a proteger lealtades.

- Enfrentar la diferenciación de culturas como tendencia laboral, producto de organizaciones que operan en entornos globales.

- Asumir una gestión humana con criterios globales, lo cual amerita apropiarse y manejar rasgos culturales diferenciados, que superan el ámbito local.

- Administrar los intangibles: talentos, valores, creencias y compromisos entre otros.

En términos más simples, se está en presencia de una realidad organizacional emergente, multicausal, polivalente, cambiante, consensual y centrada en el valor del conocimiento y los determinismos culturales, por lo cual la visión de la gestión humana debe trascender el plano simplista y homogéneo, para asumir el reto de moverse en la complejidad, incertidumbre y diversidad.

\section{La Gestión Humana, un puntal clave para el diagnóstico de la cultura y la administración de la diversidad y pluriculturalismo.}

Una de las evidencias actuales de la complejidad en el rol gerencial en el contexto de lo humano, tiene que ver con el giro de pasar de una "gestión respon- 
El reto de la gestión humana frente a la complejidad y pluralidad cultural Rodríguez Pérez, María Candelaria

sabilizada de actividades transaccionales enmarcado estrictamente en lo administrativo, como lo es la selección de personal, la administración de beneficios, la formación, el desarrollo y atención a las preocupaciones de los empleados, al plano de la función consultora sobre estrategia empresarial, asesoramiento y gestión del cambio" (Butteriss, 2001:66). En otras palabras, "los sistemas de administración de Recursos Humanos están pensados con un enfoque de cierta homogeneidad(carácter local), dentro de la heterogeneidad en cuanto a valores, idiosincrasia, cultura y lugar físico de los empleados" (López y Ponce, 1999:220).

Para ilustrar esta realidad, se presenta un panorama en donde "para muchas compañías $3 / 4$ partes de su ganancia estén generadas en el exterior, con personal que habla otros idiomas, en otras culturas y que requieren crecientes niveles de interacción transcultural" (López y Ponce, 1999:220). En términos más simples, se esta en presencia de un "fuerza de trabajo diversa formado por personas con diferentes cualidades o que pertenecen a diferentes grupos culturales" (Daft y Marcic, 2005:341).

En contraste, Dave Ulrich citado por Butteris (2001:69), plantea que la gestión de recursos humanos debe responder a los requisitos cambiantes, con cuatro claves, la primera, orienta dicha gestión a un plano estratégico, en donde Recursos Humanos actúa como un socio estratégico y es responsable de alinear su gestión con la estrategia empresarial para facilitar la ejecución de la misma. La segunda clave se centra en la gestión de la infraestructura de Recursos Humanos donde actúa de experto administrativo y desarrolla la reingeniería de los procesos de organización del factor humano. La tercera clave, compromete la gestión de Recursos Humanos con la contribución que la misma debe tener con una actuación en defensa y respuesta a las necesidades del empleado, a fin de incrementar su compromiso y capacidad.Por último, juega un papel importante en la gestión de la transformación y el cambio organizacional, a fin de crear una organización renovada. Consecuentemente supone que se pueda enfrentar los cambios a través de estrategias adaptativas acorde a las exigencias de la globalización y el desarrollo e incorporación de tecnología.

Por lo tanto, se determinan cambios en los roles originarios de esta disciplina, hacia una reorientación que redimensiona y enaltece la gestión de lo humano, ubicando otros escenarios en los cuales amerita una intervención permanente. Por ende, el papel de socio estratégico y agente de cambio, determina la necesidad de impulsar cambios culturales y administrar la diversidad en la organización, para hacer frente a los procesos tecno-estructurales en donde el impacto social amerita de una gestión de cara a las personas (empleados).

Asimismo, Butteriss (2001) plantea crear un sistema de valores comunes para toda la empresa, que mantenga unidas las muchas unidades de negocio y les proporcione una dirección. Por tanto, permite considerar la idea señalada con anterioridad referida a la "cultura fusionada", en donde puedan crearse puentes a pesar de la diversidad y diferenciación presentes en la organización desde el plano de sus áreas de funcionamiento, y por otro lado, lo atinente a las individuali- 
dades de los actores inmersos en el proceso.

Por consecuencia, supone identificar las actitudes de los individuos en la organización, para evaluar la cultura actual de la organización, la cultura futura deseada, la diversidad de culturas presentes y sus predominios, de manera que se determine la brecha que existen entre estas y establecer las acciones que integren las múltiples formas de encarar el cambio cultural y la diversidad que éste presente.

Es importante entender, que las organizaciones presentan características culturales propias, las cuales parten del proceso de construcción de realidades en respuesta a los objetivos organizacionales, por tanto, crean un conjunto de relaciones sociales y dan paso a la integración de características culturales que son el reflejo de la realidad nacional y mundial. En consecuencia, se genera una complejidad cultural que busca como mecanismo de intermediación crear una actitud tendiente al pluriculturalismo, sobre la base de un enfoque administrativo y de gestión organizacional que considere la diversidad.

Por estas razones, se entienden los procesos organizacionales desde la concepción de sistema social. Por tanto suponen estar dotados de procesos de interacción, en donde se hacen presentes normas y estructuras que tienen características sobre las cuales se configuran en su interior el llamado sistema cultural, cuyo objetivo, es el mantenimiento y desarrollo de la institución, a través de un proceso de formación de componentes ideacionales formados por esquemas colectivos de significados, valores, conocimientos y creencias, sustentados por los elementos estructurales de la organización, entre los cuales se encuentran los objetivos, las estrategias, mecanismos de control y políticas (Allaire y Firsirotu, 1992:30). De manera que estos componentes permiten a las organizaciones, como sistemas socioculturales, diferenciarse en base a las características de la cultura, las cuales se traducen en el carácter particular de cada organización.

\section{Algunos aspectos a considerar en la Administración de la diversidad y pluralidad cultural por parte de la gestión Humana.}

En virtud de todos los argumentos abordados hasta ahora, se esbozaran algunos aspectos a considerar en la administración de la diversidad y pluralidad cultural por parte de la gestión humana a nivel de las organizaciones globales.

Los retos planteados para una Gestión Humana orientada a gestionar la diversidad implican ciertas acciones las cuales se pueden concretar en las siguientes:

1. Producto de la diversidad en los grupos que laboran en una organización, es común observar acciones orientadas por criterios excluyentes, etnocentristas, estereotipados o cargados de prejuicios, por lo cual, el gran reto de la gestión humana frente a la diferenciación es lograr acciones que permitan "igualdad de oportunidades en el seno de la organización" (kreitner y Kinicki, 1997:75).

2. La conscientización y sensibilización orientada al manejo de la diversidad, es un gran reto en la gestión humana, 
El reto de la gestión humana frente a la complejidad y pluralidad cultural Rodríguez Pérez, María Candelaria

en tanto que permite lograr acciones responsables en pro de la inclusión y el pluriculturalismo, en consecuencia se toman a los mejores trabajadores sin menoscabo por causa de acciones excluyentes por raza, sexo, edad, formación, habilidad, clases sociales entre otras.

3. Al lograr el equilibrio y la equidad producto del manejo de la diversidad, es necesario incorporar esta perspectiva a los procesos y sistemas de Recursos Humanos, en las políticas de selección, remuneración y capacitación entre otros.

4. Se debe potenciar un liderazgo capaz de llevar a la práctica las políticas de pluralismo y administración de la diversidad, para lograr propiciar el desarrollo pleno de los trabajadores al interno de la organización.

5. Redimensionar el rol de la gestión humana, hacia una función de carácter estratégica bajo una configuración del trabajo más diferenciada, lo cual amerita administrar lealtades, necesidades y culturas diversas.

6. Lograr una gestión humana con gran sensibilidad transcultural, capaz de seleccionar, mantener, formar y retribuir bajo esquemas pluriculturales y de sensibilidad cultural. Sin embargo, la tarea no es fácil, ya que hay que vencer obstáculos, como el que se da, producto del choque cultural que se origina por la incapacidad que tiene el trabajador de adaptarse a un entorno cultural diferente y así mismo, el choque cultural inverso que se da, cuando el expatriado retorna a su país de origen.
7. Otro aspecto a considerar, es el que emerge del relativismo cultural, en el cual las diferencias culturales discrepan en la definición de lo bueno y lo malo. Por este motivo, la gestión de la diversidad debe lograr el conocimiento de las distintas culturas que confluyen en una misma organización, sin perder de vista la cultura local del país y región en donde opera la empresa matriz y sus filiales. Dicho conocimiento, permitiría desarrollar estrategias cónsonas a las configuraciones culturales que confluyen en una organización multicultural.

8. Lograr administrar la diversidad, amerita además, lograr la fusión de una cultura organizacional más o menos definida, respetando las diferencias culturales y acoplándolas a las exigencias de la casa matriz, sin atropellar las identidades e idiosincrasias del país de origen del expatriado y de los trabajadores locales en donde operan las filiales. Esto requiere de una "gestión con sensibilidad cultural, mente abierta y gran intuición" (Gómez, et al., 2000:518).

9. Destacar la necesidad de administrar empleados de un país que vive y trabaja en otro país (expatriado) (Gómez et al., 2000:500). Esto trae consigo ventaja y desventajas que hay que saber administrar y canalizar por parte de una gestión de Recursos Humanos bajo un enfoque global.

10. Lograr una gestión humana que se maneje bajo los parámetros de la heterogeneidad, complejidad, consenso, integración y descentralización.

11. Adoptar un enfoque plural que propicie una cultura permeable, participati- 
va y flexible que dé la apertura necesaria para propiciar un ambiente de trabajo favorable.

12. Superar la perspectiva mecanicista del hombre como apéndice de la máquina, autómata y limitado en su creatividad, para entrar en un enfoque que asume una posición que reconoce la esencia humana, la capacidad creativa, el talento humano, el conocimiento como intangible inalienable del trabajador, marcando un reto sin precedente en la necesidad de repensar el rol de la gestión humana.

13. El fin último del manejo de la diversidad por parte de la Gestión Humana, es lograr una actitud multicultural al interno de la organización, a través de la capacitación de los empleados y directivos, a fin de que la persona logre integrar las diferencias y se adapte tanto desde el punto de vista cognoscitivo como del comportamiento (Daft y Mercic, 2005).

\section{A manera de conclusión}

La complejidad forma parte del paradigma emergente, en el cual se trata de dar explicación a las nuevas realidades sociales signadas de incertidumbre, diferenciación, caos, inestabilidad y crisis. Como resultado, se genera un impacto en los supuestos que han prevalecido, quedando superados por otras formas de explicar los escenarios emergentes (sistemas, relaciones y estructuras, entre otros).

Es así, como la complejidad presente en el sistema social, configura una mayor diversidad y pluralidad en el plano cultural y por ende impacta a las organizaciones y su cultura interna.
Por ello, no se puede perder de vista las actitudes que surgen como expresión de las identidades de los miembros de un determinado grupo, en relación a acciones tendientes a la exclusión, etnocentrismo, intolerancia y discriminación, siendo estas las formas a través de las cuales se percibe la naturaleza de la cultura que impera en la organización y por tanto atenta contra la aceptación de la pluralidad y diversidad.

Por ello, la pluralidad denota la tendencia a nivel de las organizaciones, en la cual se admite al interno de una cultura determinada, la coexistencia de subculturas que nutren de nuevos valores, ideologías y principios. Por lo tanto, uno de los factores que inciden en la pluralidad es la diversidad, la cual en su dimensión básica se clasifica en la diferenciación dada por la raza, religión, etnicidad, género, edad, habilidad física, entre otras.

Por consecuencia, se necesita repensar el rol de la Gestión Humana, desde una orientación que amerita administrar la diversidad con una actitud pluralista, abierta, flexible y de gran sensibilidad transcultural, orientada a la búsqueda de la igualdad de oportunidades, el trato digno del Recurso Humano y sobre todo el respeto por las diferencias. Para ello, hay que capacitar en materia de diversidad.

\section{Referencias Bibliográficas}

Agudelo, M.G. y Alcalá, R.J. (2003). La Complejidad. [Documento en línea] disponible en www.redcientifica.com [consulta Octubre 2004].

Allaire, Yvan y Firsirotu, Mihaela (1992). Teorías sobre la cultura organizacional. En Abravanel Harry et al. (Ed) Cultura 
El reto de la gestión humana frente a la complejidad y pluralidad cultural

Rodríguez Pérez, María Candelaria

Organizacional. Aspectos teóricos, prácticos y metodológicos. Editorial Legis. Santa fé de Bogotá, Colombia, pp. 3 -33.

Arias, Fernando y Heredia, Víctor (2000). Administración del Recurso Humano para el alto desempeño. Editorial Trillas. México, pp.287-293.

Butteriss, Margaret (2001). Reinventando Recursos Humanos. Cambiando los roles para crear una organización de alto rendimiento. Editorial Edipe. España, pp. 65-80.

Certo, Samuel (2001). Administración Moderna. Prentice Hall. Colombia.

Daft, R. y Marcia, D. (2005). Introducción a la Administración. Editorial Thomson. México.

Etkin, J. y Schvarrstein, L. (1997). Identidad de las organizaciones. Invarianza y cambio. Editorial Paidos. México.

Gómez, Luis; Balkin, David y Cardy, Robert (2000). Gestión de Recursos Humanos. Prentice may. España.

López, Gustavo y Ponte, Jorge (1999). El nuevo papel de Recursos Humanos. En Andersen Arthur (Ed). El management en el siglo XXI. Herramientas para los desafíos empresariales de la próxima década. Editorial Granica. México, pp. 217-243.

Morin, Edgar (1998). Introducción al Pensamiento Complejo. Editorial Gedisa. España.

Morin, Edgar (1995). Sociología. Editorial Tecno. España, pp.141-149.

Navarro, P. (1996). El fenómeno de la Complejidad Social Humana. Universidad de Oviedo, disponible http:// www.netcom.es/pnavarro/ Publicaciones/ComplejidadSocial.html - 101k [Consulta: 2004, Noviembre 05]

Kreitner, Robert y Kinicki, Angelo (1997). Comportamiento de las organizaciones. Mc Graw. Hill. España, pp. 55-74.

Stoner, James; Freeman, Edgard y Gilbert, Daniel (1996). Administración. Sexta Edición. México, pp.198 - 220.

UNESCO (2002). Declaración universal sobre la diversidad cultural. Disponible http// www.unesco.org/culture/pluralism/diversity/html_sp/index_sp.shtml [Consulta 2004, Diciembre 16]. 